\title{
BMJ Open Evidence-based occupational health and safety interventions: a comprehensive overview of reviews
}

\author{
Birgit Teufer (1D) , Agnes Ebenberger, Lisa Affengruber, Christina Kien, Irma Klerings, \\ Monika Szelag, Ludwig Grillich, Ursula Griebler
}

To cite: Teufer B, Ebenberger A, Affengruber $\mathrm{L}$, et al. Evidencebased occupational health and safety interventions: a comprehensive overview of reviews. BMJ Open 2019;9:e032528. doi:10.1136/ bmjopen-2019-032528

- Prepublication history and additional material for this paper are available online. To view these files, please visit the journal online (http://dx.doi. org/10.1136/bmjopen-2019032528).

Received 22 June 2019

Revised 05 November 2019

Accepted 21 November 2019

Check for updates

(C) Author(s) (or their employer(s)) 2019. Re-use permitted under CC BY-NC. No commercial re-use. See rights and permissions. Published by BMJ.

Department for Evidence-based Medicine and Evaluation, Danube University Krems, Krems, Austria

Correspondence to

Ms Birgit Teufer;

birgit.teufer@donau-uni.ac.at

\section{ABSTRACT}

Objectives 0ccupational injuries and diseases are a huge public health problem and cause extensive suffering and loss of productivity. Nevertheless, many occupational health and safety $(\mathrm{OHS})$ guidelines are still not based on the best available evidence. In the last decade, numerous systematic reviews on behavioural, relational and mixed interventions to reduce occupational injuries and diseases have been carried out, but a comprehensive synopsis is yet missing. The aim of this overview of reviews is to provide a comprehensive basis to inform evidence-based decision-making about interventions in the field of $\mathrm{OHS}$. Methods We conducted an overview of reviews. We searched MEDLINE (Ovid), the Cochrane Library (Wiley), epistemonikos.org and Scopus (Elsevier) for relevant systematic reviews published between January 2008 and June 2018. Two authors independently screened abstracts and full-text publications and determined the risk of bias of the included systematic reviews with the ROBIS (Risk of Bias in Systematic Reviews) tool.

Results We screened 2287 abstracts and 200 full-texts for eligibility. Finally, we included 25 systematic reviews with a low risk of bias for data synthesis and analysis. We identified systematic reviews on the prevention of occupational injuries, musculoskeletal, skin and lung diseases, occupational hearing impairment and interventions without specific target diseases. Several interventions led to consistently positive results on individual diseases; other interventions did not show any effects, or the studies are contradictory. We provide detailed results on all included interventions.

Discussion To our knowledge, this is the first comprehensive overview of behavioural, relational and mixed interventions and their effectiveness in preventing occupational injuries and diseases. It provides policymakers with an important basis for making evidence-based decisions on interventions in this field. PROSPERO registration number CRD42018100341

\section{BACKGROUND}

Occupational injuries and diseases cause extensive suffering and loss of productivity. The WHO estimates that, globally, there are 1.2 million deaths per year attributable to occupational risks, which relates to $2.1 \%$ of all deaths in the general population. ${ }^{12}$ Estimates from the Workplace Safety and Health

\section{Strengths and limitations of this study}

To our knowledge, this is the first comprehensive overview of reviews on behavioural, relational and mixed interventions to prevent injuries at work and occupational diseases.

- We based our overview of reviews on an extensive, comprehensive and systematic literature search.

- Two scientists independently carried out all the essential steps in the preparation of this review.

- A secondary literature analysis may result in evidence base gaps, either due to periods not covered by the included systematic reviews (SRs) or to further limitations in the SRs.

- We considered only SRs with a low risk of bias for the data extraction analysis to ensure validity but on the contrary, this approach may have led to a loss of information in topics where only SRs with a high or unclear risk of bias were available.

Institute, Singapore, in cooperation with the International Labour Organization (ILO) are even higher, with nearly 2.8 million deaths annually being attributed to work, and another 374 million to non-fatal occupational accidents. ${ }^{3}$ Although the estimation of occupationally related mortality and morbidity worldwide varies widely due to methodological problems, the general conclusion is that occupational diseases and injuries are a huge public health problem. ${ }^{4}$ Not only do social and ethical arguments support preventive occupational health and safety (OHS) services ${ }^{5}$ but so do the monetary consequences of ill health at work. ${ }^{6}$

Decisions on which interventions to implement are usually dominated by negotiations between unions, employers and government representatives. ${ }^{7}$ However, expert advice can be seriously biassed, ${ }^{8}$ leading to wide variations in expert judgements. ${ }^{9}$

The WHO states that the principle that all their guidelines must be based on systematic and comprehensive assessment of potential benefits and harms. ${ }^{10}$ Nevertheless, many 
OHS guidelines are still not based on the best available evidence. ${ }^{11}$ Healthcare providers and policy-makers are confronted with an unmanageable amount of information, ${ }^{12}$ and there is a large amount of systematic reviews on interventions to prevent single occupational diseases or injuries according to very specific risks available (eg, on work.cochrane.org). Systematic reviews are regarded as the most appropriate method to avoid bias in synthesising the best available evidence. Because so many systematic reviews are already available, we conducted an overview of reviews. That means we compiled the results from multiple systematic reviews (SRs), addressing the effects of interventions for a health problem or condition according to a predefined procedure. We appraised their quality and summarised their evidence for important outcomes. ${ }^{13}$ The aim of this overview of reviews is to provide a comprehensive basis for making evidence-based decisions on interventions in the OHS field by answering the following research question:

'What effects do interventions in the workplace setting have on working conditions, exposure to disease-causing factors and the behaviour of employees as well as on accidents at work and the development of occupational diseases?'

To our knowledge, this is the first comprehensive overview of reviews on behavioural, relational and mixed interventions to prevent injuries at work and occupational diseases, based on a comprehensive and systematic search, critical appraisal and the synthesis of SRs. It enables prioritisation between different interventions based on the quality of evidence (QoE).

\section{METHODS}

We have registered the protocol of the overview of reviews at the International Prospective Register of Systematic Reviews (PROSPERO). We adhered to the Preferred Reporting Items for Systematic Reviews and Meta-Analyses (PRISMA) statement ${ }^{14}$ throughout this manuscript (PRISMA checklist see online supplementary appendix 1).

\section{Study design}

We conducted an overview of reviews following the guidance provided in the Cochrane Handbook. ${ }^{12}$

\section{Information sources and literature search}

An information specialist conducted the database search in MEDLINE (Ovid), the Cochrane Library (Wiley), epistemonikos.org and Scopus (Elsevier) in June 2018. The usefulness of SRs also depends on their actuality, but there is no consensus on when SRs are obsolete and when an update is necessary. ${ }^{15}$ To prevent us from relying on outdated evidence, we limited the search to SRs published since 2008. The full search strategies are reported in online supplementary appendix 2.

Additionally, we checked the bibliographies of the included SRs and relevant articles for further references to eligible reviews. To ensure that the evidence is up to date, we conducted forward citation tracking of selected SRs using Scopus (Elsevier). We also checked the websites of the Cochrane Work Group (https://work.cochrane. org/cochrane-reviews-about-occupational-safety-andhealth), the ILO (https://www.ilo.org/global/langen/index.htm), the Occupational Safety and Health Administration (OSHA) (https://www.osha.gov/pls/ publications $/$ publication.AthruZ?pType=Types), the WHO (http://www.who.int/occupational_health/publications/en/) and the European Agency for Safety and Health at Work (EU-OSHA) (https://osha.europa.eu/ en/tools-and-publications).

\section{Eligibility criteria}

You can find a detailed description of the inclusion and exclusion criteria in table 1 . We provide additional information and definitions thereafter.

We defined systematic review according to the Cochrane Handbook as 'a literature review that attempts to collate all empirical evidence using (a) clearly stated objectives and predefined eligibility criteria, (b) an explicit reproducible methodology, (c) a systematic search, (d) an assessment of the validity of the findings of the included studies and (e) a systematic presentation, and synthesis, of the characteristics and findings of the included studies'. ${ }^{12}$ In addition, to be included in this overview of reviews, SRs had to conduct the search in at least two scientific databases and perform abstract and full-text screening by two independent reviewers.

We included SRs of all types of workplace-related interventions designed to protect against occupational injuries and for the primary prevention of occupational diseases, including legislation and audits by the health and safety executive as well as organisational-level workplace interventions. According to Montano et al, ${ }^{16}$ interventions that modify working conditions can be described in three broad categories: material condition (physical and chemical agents needed during work), work time-related condition (amount of working time and intensity of work) and work organisation conditions (psychological factors and processes and procedures necessary for the completion of work tasks).

We defined occupational diseases in accordance with the definition of the ILO ${ }^{17}$ that groups occupational diseases caused by exposure to agents arising from work activities (caused by chemical agents, physical agents, biological agents or infectious or parasitic diseases), by target organ systems (respiratory diseases, skin diseases, musculoskeletal disorders and mental and behavioural disorders) and occupational cancer.

Work-related injuries are injuries which are causally, locally and temporally related to the insured occupation and which lead to physical injury. ${ }^{18}$ Risk factors that can lead to occupational injuries or diseases were defined as changes in environmental conditions, changes in exposure to disease-causing factors (eg, noise, extreme 
Table 1 Eligibility criteria for the overview of reviews on OHS interventions

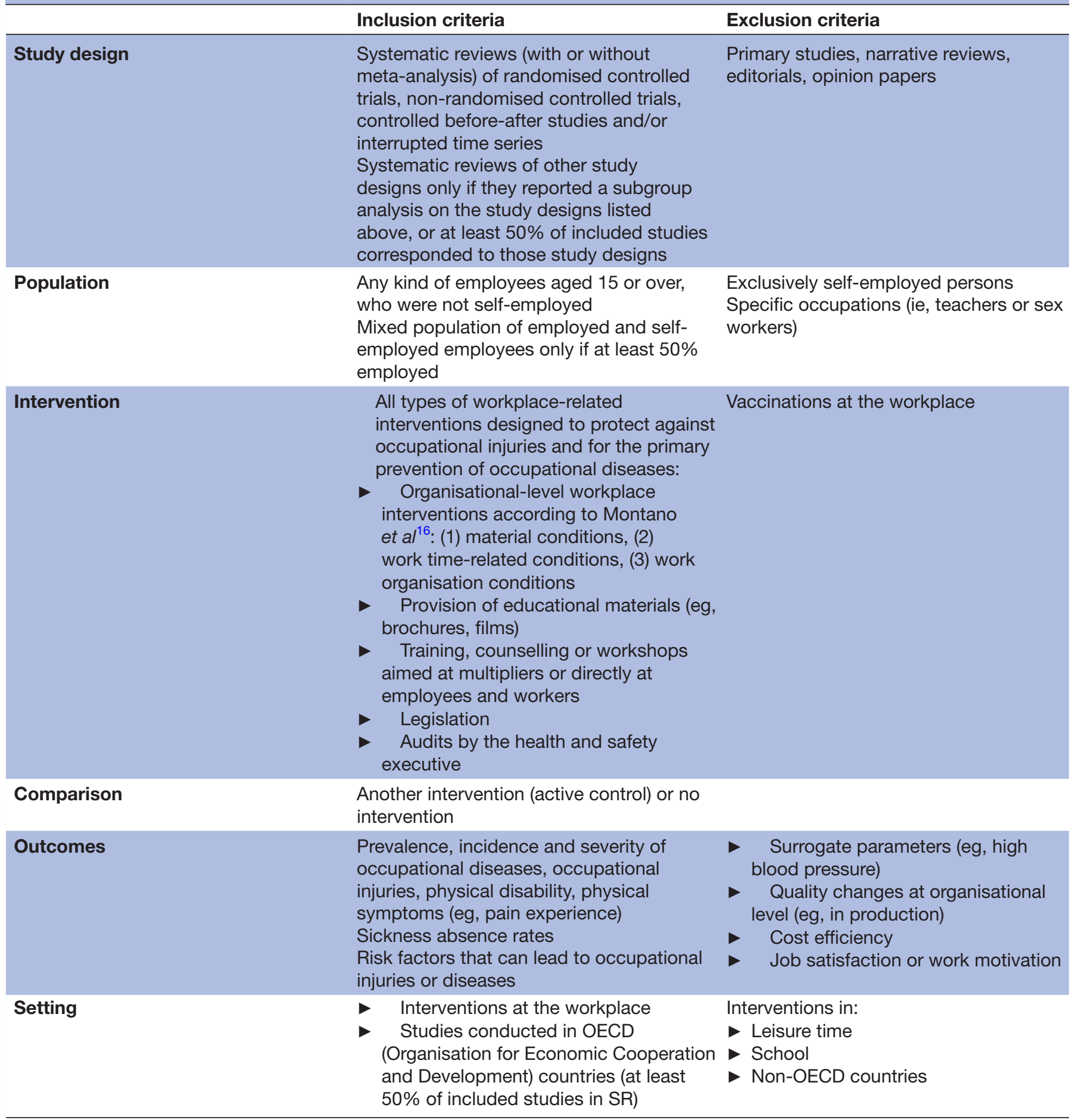

OHS, occupational health and safety; SR, systematic review.

temperatures) and changes at the employees' or workers' behavioural level (eg, wearing protective equipment).

\section{Study selection}

The reviewer team consisted of five persons with experience in conducting systematic reviews (BT, AE, LA, UG, MS). Each study was independently assessed by two reviewers from this team in two consecutive steps (abstract and full-text selection) based on the previously defined inclusion criteria. Conflicts between reviewers were resolved through discussion and consensus or by involving a third person from the reviewer team. We used the software Covidence (https://www.covidence.org/) for the study selection process.

\section{Risk of bias assessment and certainty of evidence}

Two independent reviewers appraised the quality of the SRs with the ROBIS (Risk of Bias in Systematic Reviews) 
Table 2 Definition and interpretation of risk of bias ${ }^{19}$

\begin{tabular}{ll}
\hline Risk of bias & Interpretation \\
\hline Low risk of bias & $\begin{array}{l}\text { The findings of the review are likely to be reliable. No concerns with the review process, or } \\
\text { concerns were appropriately considered in the review conclusions. The conclusions were } \\
\text { supported by the evidence and included consideration of the relevance of included studies. }\end{array}$ \\
High risk of bias & $\begin{array}{l}\text { One or more of the concerns raised during the assessment was not addressed in the review } \\
\text { conclusions, the review conclusions were not supported by the evidence or the conclusions did } \\
\text { not consider the relevance of the included studies to the review question. }\end{array}$ \\
Unclear risk of bias & There is insufficient information reported to make a judgement on risk of bias.
\end{tabular}

tool. ${ }^{19}$ This assesses four dimensions of SRs: 'study eligibility criteria', 'identification and selection of studies', 'data collection and study appraisal' and 'synthesis and findings'. The result is an assessment of the risk of bias of each SR using the categories low, unclear and high (see table 2). Disagreements in appraisal between reviewers were resolved through discussion and consensus or by involving a third person.

\section{Data synthesis and analysis}

We synthesised data narratively and in forms of evidence tables. Due to the large number of SRs available and to ensure validity, we excluded SRs with a high or unclear risk of bias for our data synthesis and analysis.

For SRs with a low risk of bias we extracted the following data:

- Details of the SR (author, title, year of publication, aim of the SR)

- Details of the included studies (number of studies and persons included, risk of bias of studies)

- Details of the population (age, gender, type of occupation)

- Details of the intervention (duration, type of measures)

- Details of the results (time of outcome measurement, results for each endpoint)

- Quality of evidence (if reported in the included SR) Several institutions (eg, Cochrane, WHO, BMJ Clinical Evidence and many more ${ }^{20}$ ) and the researchers of several included SRs use the GRADE approach (Grading of Recommendations, Assessments, Developments and Evaluations) or modifications thereof to assess the QoE.
Table 3 presents the significance of the four levels of evidence.

If the researchers of the included SRs used evidence assessment tools other than the GRADE approach, we provide the definition of the used levels of evidence in a footnote.

We did not extract data from primary studies. If information was not apparent from the included review, it was presented as 'not available' (n.a.). If the SR was an update of an older version, we only extracted data of the most recent version. We did not find SRs that answered the exact same research question; therefore, we did not check for overlap in the included primary studies.

\section{Patient and public involvement}

There were no funds or time allocated for patient or public involvement so we were unable to involve patients. If, after consultation with the sponsor, further dissemination of the results takes place, we will invite representatives of the public to help us write a plain language summary.

\section{RESULTS}

We identified 2215 citations from electronic database searches after the removal of duplicates, and we found another 72 citations from additional searches (reference list checking and forward citation tracking). All potentially relevant SRs on the searched web pages (see 'Information sources and literature search') were crosschecked with hits from the previous database search. Because the database search had already covered all relevant SRs, we did not identify any new references in this

Table 3 Significance of the four levels of evidence ${ }^{51}$

\begin{tabular}{ll}
\hline Quality level & Definition \\
$\begin{array}{l}\oplus \oplus \oplus \oplus \\
\text { high }\end{array}$ & We are very confident that the true effect lies close to that of the estimate of the effect \\
$\oplus \oplus \oplus 0$ & $\begin{array}{l}\text { We are moderately confident in the effect estimate: the true effect is likely to be close } \\
\text { to the estimate of the effect, but there is a possibility that it is substantially different }\end{array}$ \\
$\begin{array}{ll}\oplus \oplus 0 \mathrm{l} \\
\text { low }\end{array}$ & $\begin{array}{l}\text { Our confidence in the effect estimate is limited: the true effect may be substantially } \\
\text { different from the estimate of the effect }\end{array}$ \\
$\begin{array}{l}\oplus 00 \\
\text { very low }\end{array}$ & $\begin{array}{l}\text { We have very little confidence in the effect estimate: the true effect is likely to be } \\
\text { substantially different from the estimate of effect }\end{array}$ \\
\hline
\end{tabular}




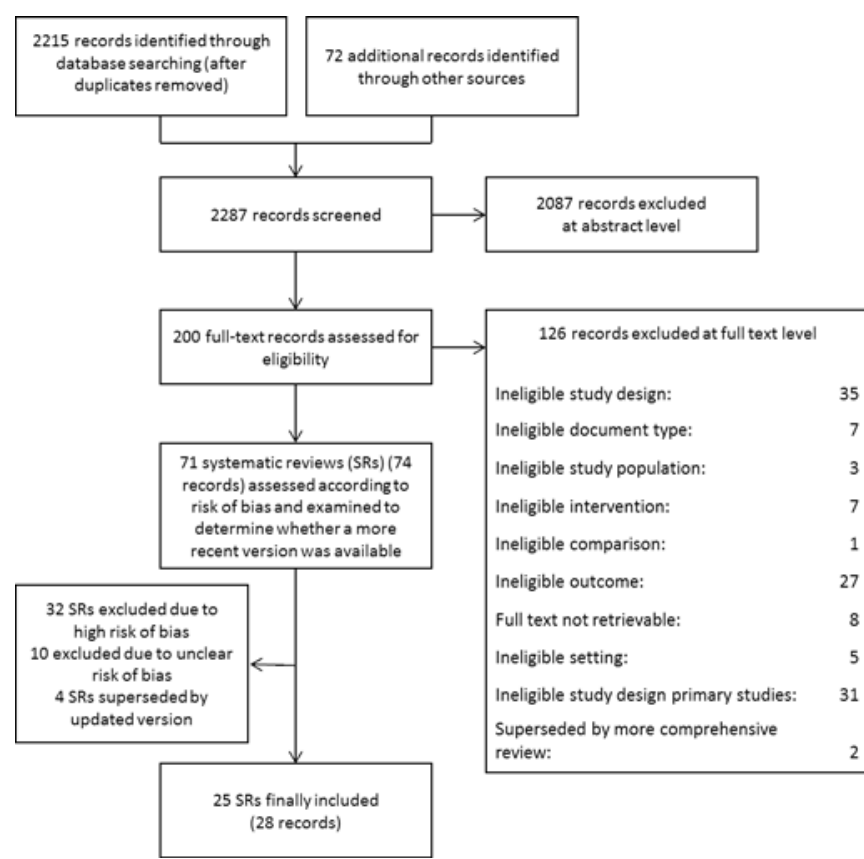

Figure 1 Preferred Reporting Items for Systematic Reviews and Meta-Analyses flowchart of the study selection process.

step. Overall, 2287 citations were screened by title and abstract and, subsequently, we assessed 200 full-texts for eligibility. From the 71 SRs ( 74 records) that met our eligibility criteria, we appraised 32 with a high risk of bias and 10 with an unclear risk of bias. Four of the included SRs were updates from previous versions. Finally, we included 25 SRs reported in 28 publications for data synthesis and analysis. Figure 1 shows the details of the study selection process. We provide a list of excluded full-text articles with reasons for exclusion as well as a detailed risk of bias assessment (including SRs with a high or unclear risk of bias) in online supplementary appendices $3 ; 4$.

\section{Description of included studies}

Online supplementary appendix 5 provides an overview of the included SRs, summarising the interventions, description of measured outcomes and risk of bias rating.

From the 25 included SRs, a considerable number of studies (12 SRs) dealt with research questions on the topic of the prevention of musculoskeletal disorders, seven investigated the efficacy of interventions for the prevention of occupational injuries and three reviews studied interventions for the prevention of occupational skin and lung diseases. One review examined the efficacy of interventions for the prevention of occupational hearing loss, and another two SRs dealt with diverse interventions about OHS without limiting to a specific target disease. Detailed information about all above mentioned SRs including the interventions, control interventions, included studies, setting, method of data synthesis and a graphical presentation of the results can be found in the online-only supplementary material (online supplementary appendix 5).

\section{Prevention of musculoskeletal disorders}

Overall, 12 SRs reported on different interventions for the prevention of musculoskeletal disorders. They included mixed interventions with several different components, ${ }^{21-25}$ physical exercises at the workplace, ${ }^{26}$ work organisation and psychosocial working environment, ${ }^{27}$ educational interventions for the prevention of musculoskeletal disorders, ${ }^{28}$ ergonomic interventions ${ }^{29}$ and interventions in the area of manual handling of loads. ${ }^{30-32}$

Strengthening exercises or fitness training had a positive effect on musculoskeletal disorders in general as well as in the shoulder and neck area and on back pain in various occupational groups. ${ }^{22} 2426$ The QoE varied widely between outcomes. See online supplementary appendix 6 for further details.

Lowry et $a t^{24}$ found a significant reduction in the prevalence of shoulder pain with workplace adjustments (QoE: low). Additional breaks compared with conventional break schedules seem to reduce symptom intensity in different body regions (QoE: moderate). ${ }^{27}$ Both SRs included a wide range of occupational groups.

Educational interventions alone (eg, training) showed no effect on the reduction of musculoskeletal disorders (QoE: very low to moderate) ${ }^{25272832}$ (only on training for manual material transfer).

The results of ergonomic interventions on musculoskeletal disorders are mixed and varied but, in general, tend to result in some form of pain reduction. Chen et $a l^{22}$ found evidence of low quality for the efficacy of ergonomic interventions on neck pain. The use of an arm support with alternative computer mice reduces the incidence of musculoskeletal disorders in the neck/shoulder but not in the right upper extremity (QoE: moderate) ${ }^{29}$ There is no difference for musculoskeletal disorders in the neck/shoulder and right upper extremity between alternative and conventional computer mice with and without arm support (QoE: moderate). ${ }^{29}$ Richardson et $a l^{25}$ found a positive effect of unstable shoes on pain in nurses (QoE: n.a.). No effect of physiotherapist or ergonomist feedback sessions on the optimal design of computer workstations, work techniques and the psychosocial aspects of work could be observed (QoE: low) ${ }^{27}$ Goodman et $a t^{23}$ concluded that not a single measure but a combination of measures (included interventions, for example, education, work station adjustments, exercise, rest breaks, specific ergonomic equipment) is most effective in addressing cumulative trauma disorder symptoms.

Aids for patient transfer (both small aids such as bed steps, anti-slip mats, etc, and mechanical aids such as mechanical transport devices for patients) led to positive effects on pain and/or injuries of the musculoskeletal system in two SRs (QoE: very low to low). ${ }^{30} 31$ Stock et $a l^{27}$ showed that 'lifting programmes' as well as multicomponent interventions on safe patient handling in hospitals had no effect on several outcomes measured (eg, the prevalence of neck/shoulder pain, forearm/ wrist pain, lower back pain and musculoskeletal pain in any body region; upper extremity or back-related 
functional status; musculoskeletal work injury rates and time loss injury rates; QoE: very low to low) except for two outcomes: they found low-quality evidence that a safe lifting programme is more effective than usual practice in reducing the frequency of work-related shoulder pain and work-related low back pain (QoE: low) ${ }^{27}$

\section{Prevention of occupational injuries}

Overall, seven SRs investigated interventions for the prevention of occupational injuries. One review dealt with different interventions for the prevention of occupational injuries in the agricultural sector, ${ }^{33}$ one SR examined the effects of interventions for the prevention of occupational injuries in the construction industry ${ }^{34}$ and another review examined the effects of alcohol and drug screening of professional drivers on accidents. ${ }^{35}$ Four SRs searched for safety products and practices in the health sector to prevent occupational injuries. ${ }^{36-39}$

Rautiainen $e t a l^{33}$ found no effect of education on the prevention of injuries in the agricultural sector. Financial incentives (insurance premium discounts) showed a short-term positive effect but no long-term progressive improvement. Legislation banning endosulfan pesticides showed a progressive reduction in deaths by poisoning. Regulations for the use of rollover protection structures showed contradictory results. For all outcomes, no QoE was stated.

Van der Molen $e t a \hat{l}^{4}$ found contradictory evidence on the impact of regulations and inspections to prevent injuries in construction workers. Regional safety campaigns, training, inspections or the introduction of occupational health services are unlikely to reduce the number of nonfatal injuries in construction companies, while companyoriented measures, such as safety campaigns, a drug workplace programme or subsidies for safe scaffolding, can have a positive effect (QoE: very low for all outcomes).

Cashman $e t a l^{85}$ investigated the effects of alcohol and drug screening of occupational drivers on accidents and injuries. This review included two interrupted time series (ITS) studies which analysed data over a period of 13 and 14 years, respectively. Binding alcohol tests brought with them fewer accidents in the short-term but had no effect on the long-term trend. With regard to mandatory drug tests, the studies did not show a uniform picture of the short-term effects but a uniform strengthening of the long-term trend towards declining accident rates. The authors of the study judged the QoE as limited, which was defined as 'one low quality RCT or one CBA study or one ITS'. ${ }^{35}$

Four SRs investigated for safety products and practices in the health sector to prevent occupational injuries. Parantainen $e t a l^{\beta 7}$ showed that the use of blunt surgical suture needles reduced the risk of glove perforation (QoE: high) and the number of self-reported needle stick injuries (QoE: moderate) compared with sharp suture needles. Reddy $e t a \vec{P}^{8}$ found that the use of safe blood collection systems showed inconsistent effects on the number of needle stick injuries (QoE: very low). The use of safe passive intravenous systems showed a decrease in needle stick injuries and a reduction in the incidence of blood splashes (QoE: very low). However, evidence of moderate quality was found that active systems might increase exposure to blood. For safe injection devices (QoE: very low to low), the introduction of several safety products (QoE: very low) or safety containers (QoE: very low) showed inconsistent results, or there was no clear evidence of benefit. Two ITS studies showed that interrupted introduction of legislation on the use of safetyengineered devices reduced the rate of needle stick injuries among healthcare workers (QoE: moderate), whereas one ITS with low-quality evidence showed an increase in the level of needle stick injuries with gradual introduction. Evidence showed varying results in the trend over time for needle stick injury rates (QoE: very low to low). ${ }^{38}$

Mischke $e t a \hat{l}^{36}$ reported that there is moderate-quality evidence that double gloves reduce perforations and bloodstains on the skin compared with single gloves during surgery, which may mean a decrease in percutaneous exposure events. Triple gloves and the use of special gloves can further reduce the risk of glove perforations compared with double gloves made of normal material (QoE: low). Verbeek et $a l^{39}$ found very low-quality evidence that more breathable types of personal protective equipment (PPE) would not lead to more contamination with body fluids. Double gloves and the Centre for Disease Control and Prevention doffing guidelines reduced the risk of contamination with body fluids, and more active training in PPE use could reduce PPE errors and PPE doffing errors more than passive training (QoE: very low). However, the data all come from individual studies with a high risk of bias, so there is uncertainty about the estimates of the effects.

\section{Prevention of occupational skin and lung diseases}

Three SRs included studies about the efficacy of interventions to prevent occupational skin and lung diseases. ${ }^{40-42}$

Lunt et $a t^{40}$ found low positive effects of behavioural interventions at the workplace (training for behavioural changes or for influencing knowledge and attitudes about health and safety precautions) on exposure to occupational health hazards for workers exposed to dermal and respiratory hazards (QoE: n.a.). Luong Thanh $e t a t^{11}$ found evidence of low to very low quality that behavioural interventions (education and training to improve the use of respiratory protective equipment) did not largely contribute to workers using protective equipment correctly or more frequently. Bauer et $a t^{42}$ found that moisturisers used alone or in combination with barrier creams can provide clinically relevant protection against irritant hand dermatitis (QoE: low). For advanced training interventions for skin protection, the results of the individual studies varied considerably. Altogether, they showed no clinically relevant effect (QoE: very low). ${ }^{42}$ 


\section{Prevention of occupational hearing loss}

We identified one SR which examined the effect of interventions for the prevention of occupational hearing loss. ${ }^{43}$ On average, wearing hearing protection reduced noise exposure by about $20 \mathrm{~dB}(\mathrm{~A})$ (QoE: low), and more noise was attenuated with instruction on how to use hearing protection than without instruction (QoE: moderate). With regard to hearing impairment, there was no difference between ear protectors and earplugs at noise levels above $89 \mathrm{~dB}(\mathrm{~A})$ (QoE: very low). Implementing stricter legislation to protect against occupational hearing loss (multiple components, for example, prioritising technical and administrative controls, setting a threshold) led to an immediate reduction in the mean personal noise exposure in coal construction and a further positive, but statistically not significant, trend in the reduction of the noise dose (QoE: very low). Furthermore, the authors found no statistically significant differences between on-site training and information online (QoE: low), information about personal noise exposure and no information about it (QoE: low), intensive hearing loss prevention programmes (HLPP) compared with pure audiometry (QoE: moderate) and HLPP with personal noise exposure information compared with HLPP without this information (QoE: very low).

\section{General occupational health and safety interventions}

We included one SR that examined the effects of laws and regulations on occupational safety and health ${ }^{44}$ and one SR dealt with interventions for the prevention of the inability to work after sick leave. ${ }^{45}$

Mischke $e t a l^{44}$ found positive effects of compliance inspections on injuries at work (QoE: low). However, the effects only became apparent in the long-term (mean 36 and 48 months follow-up), and no statistically significant risk reductions could be observed in the short-term (mean 21 to 24 months). Inspections also had no statistically significant effect on employees' physical workload (QoE: low).

Van Vilsteren et $a t^{45}$ showed that interventions to prevent work disability in workers on sick leave shortened the time to first return-to-work of workers with musculoskeletal disorders (QoE: moderate) and the time to lasting return-to-work (Qoe: very low) for this group of workers but not for people with mental illness or cancer (QoE: very low). Workplace interventions reduced the cumulative sickness duration by an average of 33 days (QoE: high). Significant results were only shown for persons with musculoskeletal disorders but not for persons with mental illnesses. However, the risk of sick leave recurrences for persons with musculoskeletal disease was higher for workplace interventions (QoE: moderate). In addition, the authors found positive effects on the functional status of employees with musculoskeletal disorders (QoE: moderate) and pain (QoE: high) but no significant effect on depression (QoE: very low). Overall, the SR found evidence for the positive effects of workplace interventions to prevent work disability in workers on sick leave with musculoskeletal disorders (QoE: moderate) but no effects on persons with mental illnesses or cancer (QoE: low). ${ }^{45}$

\section{DISCUSSION}

This overview of reviews provides a comprehensive overview of behavioural, relational and mixed interventions and their effectiveness in preventing occupational injuries and diseases. We identified SRs on the prevention of occupational injuries, musculoskeletal, skin and lung diseases, occupational hearing impairment and interventions without specific target diseases.

Almost half of all the included reviews refer to workrelated illnesses of the musculoskeletal system, which demonstrates the importance of this topic and is in accordance with the fact that musculoskeletal disorders are one of the main causes for work-related mortality and morbidity. ${ }^{46}$ Several interventions (eg, strengthening exercises, individual ergonomic interventions and patient transfer aids) led to consistently positive results on individual musculoskeletal system diseases. Other interventions (eg, educational and cognitive behavioural interventions) targeting illnesses of the musculoskeletal system did not show any effects, or the studies are contradictory.

With regard to the prevention of occupational accidents and the reduction of exposure to risk factors, legislation and regulations as well as inspections can be effective (eg, ban on endosulfan pesticides, legislation on the use of safety-engineered devices in the healthcare sector, etc). In some cases, however, studies showed contradictory results (eg, regulations on the use of rollover protection structures) or no effects (eg, inspections in the construction sector). Financial incentives such as insurance premium discounts and subsidies for safe scaffolding showed positive effects. Company-oriented interventions such as safety campaigns, awareness-raising campaigns or drug workplace programmes appear to have positive effects on injuries at work and compliance with rules. The evidence for the effectiveness of training and education interventions, in general, is mixed and must be considered specifically by target disease or intervention.

In the medical field, there is partly good evidence for the use of safety products (eg, blunt needles, double gloves, etc), but inconsistent effects have been observed for other safety products and practices (eg, use of safe blood collection systems, safe injection needles, etc). With regard to skin and lung diseases, there is some good evidence of the efficacy of various interventions (eg, moisturisers, barrier creams, protective gloves, etc). There are also effective interventions to prevent work-related hearing loss (eg, wearing hearing protection, well-implemented HLPP).

A strength of this overview of reviews is the extensive literature search. The search strategy was not restricted to specific target diseases or interventions to obtain the most comprehensive results possible. Through the use of several additional search strategies, such as reviewing 
multiple organisational websites and backward and forward citation tracking, further SRs could be identified. Nonetheless, there remains a residual risk of not having found all relevant reviews. As a methodological limitation, it can be stated that a secondary literature analysis may result in evidence base gaps, either due to periods not covered by the included SRs or to further limitations in the SRs, such as limitations on study design or included interventions. ${ }^{47}$ Due to the overview of reviews method, we did not extract data from the individual studies included in the SRs. Therefore, we have taken over the assessment of the QoE from the authors of the reviews. Almost all included reviews used the GRADE system for the QoE evaluation and assessed the QoE dually. Therefore, we are quite confident that we can rely on the reviewers' assessment of QoE, but we are aware that these assessments may be subject to subjective influences of the respective systematic review authors. Besides further criticism of the grading systems ${ }^{48}$ there is also the danger of an undiscovered small study effect. Small study effects refer to the fact that trials with smaller sample sizes are more likely to report larger beneficial effects than large trials. ${ }^{49}$ Even the capabilities of bias identification methods, such as funnel plots, are limited when recommendations or metaanalyses are based on a limited number of small trials ${ }^{50}$ or even only one available trial. Therefore, the danger of overestimating effects of small studies and assessments of high quality of evidence remains where only a few small studies exist. The assessment of the QoE must be interpreted with particular caution in interventions where only a few studies are available. In this overview of reviews this may be observed for some ergonomics interventions for computer users, where review authors have arrived at a high or moderate quality of evidence in their assessment, although in some cases only one or two small studies were available (online supplementary appendix 6).

Our approach of considering only SRs with a low risk of bias for the data extraction analysis may be seen as ensuring validity in topics where good SRs were available. On the contrary, this approach may have led to a loss of information in topics where only SRs with a high or unclear risk of bias were available, such as mental diseases. However, the large number of identified SRs with a high or unclear risk of bias highlights the need for more reviews in the field of OHS that apply rigorous methods. A further strength of this overview of reviews is that two scientists independently carried out all the essential steps in the preparation of this review. This ensures that both the screening of the references and the assessment of the risk of bias of the included reviews minimised subjective influences.

\section{CONCLUSION}

Several examined OHS interventions led to consistently positive results on individual diseases; other interventions did not show any effects, or the studies are contradictory. Policymakers and other authorities must therefore carefully consider the interventions to which the limited resources available shall be applied. While the included reviews covered many relevant endpoints, cancer and circulatory diseases were not mentioned in any of them. On one hand, this may result from the limitation of certain primary study designs - such as randomised controlled trials (RCTs) or controlledbefore-after (CBA) studies - which are considered robust but may not be suitable to assess effects on diseases that are relatively rare and develop in the long-term, such as cancer. On the other hand, this may be interpreted as a demonstration of an important gap in the research literature, especially as cancer and circulatory diseases are two of the main causes for work-related mortality and morbidity. ${ }^{46}$

To our knowledge, this is the first comprehensive overview of reviews on interventions to prevent injuries at work and occupational diseases. It provides policymakers with an important basis for making evidence-based decisions on interventions in this field.

Acknowledgements We wish to thank Danielle Eder-Linder from the University of Continuing Education (Danube University Krems) for administrative support.

Contributors CK drafted the research protocol and BT, UG and LG provided substantial contributions to the protocol. BT coordinated the reviewing process. $\mathrm{BT}, \mathrm{AE}, \mathrm{LA}, \mathrm{UG}$ and MS contributed to the abstract and full-text screening, data extraction and risk of bias assessment of the reviews. IK developed the search strategy, performed the search and contributed to the data extraction. All authors wrote substantial parts of the first draft of the manuscript and revised it critically for important intellectual content, and all approved the final manuscript.

Funding This overview of reviews was funded by the Austrian General Accident Insurance Institution (Allgemeine Unfallversicherung, AUVA). The funding source had no role in the collection, analysis or interpretation of data.

Disclaimer The protocol allowed for the inclusion of all systematic reviews that fulfilled our eligibility criteria regardless of their quality (risk of bias). Due to the large number of available reviews, we decided to include for data extraction only systematic reviews with a low risk of bias.

Competing interests None declared.

Patient consent for publication Not required.

Provenance and peer review Not commissioned; externally peer reviewed.

Data availability statement Data are available upon reasonable request.

Open access This is an open access article distributed in accordance with the Creative Commons Attribution Non Commercial (CC BY-NC 4.0) license, which permits others to distribute, remix, adapt, build upon this work non-commercially, and license their derivative works on different terms, provided the original work is properly cited, appropriate credit is given, any changes made indicated, and the use is non-commercial. See: http://creativecommons.org/licenses/by-nc/4.0/.

ORCID iD

Birgit Teufer http://orcid.org/0000-0002-3324-0639

\section{REFERENCES}

1 Stanaway JD, Afshin A, Gakidou E, et al. Global, regional, and national comparative risk assessment of 84 behavioural, environmental and occupational, and metabolic risks or clusters of risks for 195 countries and territories, 1990-2017: a systematic analysis for the global burden of disease study 2017. The Lancet 2018;392:1923-94.

2 Wolf J, Prüss-Ustün A, Ivanov I, et al. Preventing disease through a healthier and safer workplace. Geneva: World Health Organization, 2018.

3 Hämäläinen P, Takala J, Kiat TB. Global estimates of occupational accidents and work-related illnesses 2017. World 2017;2017:3-4. 
4 Rushton L. The global burden of occupational disease. Current Environmental Health Reports 2017;4:340-8.

5 World Health Organization. Declaration on occupational health for all: Approved at the second meeting of the who collaborating centres in occupational health, Beijing, China, 11-14 October 1994. Geneva: World Health organization, 1994.

6 Stewart WF, Ricci JA, Chee E, et al. Lost productive work time costs from health conditions in the United States: results from the American productivity audit. J Occup Environ Med 2003;45:1234-46.

7 Verbeek J, Morata T, Ruotsalainen J, et al. Prevention of occupational diseases: implementing the evidence. Cochrane Database Syst Rev [Internet] 2013.

8 Michaels D. Doubt is their product, how industry's assault on science threatens your health. New York: Oxford University Press, 2008.

9 Sutherland WJ, Burgman M. Policy advice: use experts wisely. Nature 2015;526:317-8.

10 World Health Organization. Who Handbook for Guideline development. 2nd edition. Geneva: World Health Organization, 2014.

11 Verbeek J. Could we have better occupational health guidelines, please? Scand J Work Environ Health 2018;44:441-2.

12 Green S, Higgins JP, Alderson P, et al. Cochrane Handbook for systematic reviews of interventions; chapter 1: introduction training. cochrane.org/handbook: the Cochrane collaboration, 2011: 5.1.

13 Higgins J SG. Cochrane Handbook for Systematic Reviews of Interventions Version 5.1.0 [updated March 2011] Chapter 22 Overviews of reviews: The Cochrane Collaboration, 2011. Available: www.handbook.cochrane.org

14 Moher D, Liberati A, Tetzlaff J, et al. Preferred reporting items for systematic reviews and meta-analyses: the PRISMA statement. International Journal of Surgery 2010;8:336-41.

15 Moher D, Tsertsvadze A, Tricco A, et al. When and how to update systematic reviews. Cochrane Database Syst Rev 2008;3.

16 Montano D, Hoven H, Siegrist J. Effects of organisational-level interventions at work on employees' health: a systematic review. BMC Public Health 2014;14:135.

17 International Labour Organization (ILO). ILO list of occupational diseases (revised 2010), 2010. Available: http://www.ilo.org/wcmsp5/ groups/public/---ed_protect/---protrav/---safework/documents/ publication/wcms 125137.pdf [Accessed Available from].

18 Allgemeine Unvfallversicherungsanstalt (AUVA). Liste der Berufskrankheiten, Inkrafttreten: 01.01.2014, 2014. Available: https:// www.auva.at/cdscontent/load?contentid=10008.541831

19 Whiting P, Savović J, Higgins JPT, et al. ROBIS: a new tool to assess risk of bias in systematic reviews was developed. $J$ Clin Epidemiol 2016:69:225-34.

20 Green S, Higgins JP, Alderson P, et al. Cochrane Handbook for systematic reviews of interventions; Part 2; 12.2.1 the grade approach training.cochrane.org/handbook: the Cochrane collaboration, 2011: 5.1 .

21 Aas RW, Tuntland H, Holte KA, et al. Workplace interventions for neck pain in workers. Cochrane Database Syst Rev 2011;132.

22 Chen X, Coombes BK, Sjøgaard G, et al. Workplace-Based interventions for neck pain in office workers: systematic review and meta-analysis. Phys Ther 2018;98:40-62.

23 Goodman G, Kovach L, Fisher A, et al. Effective interventions for cumulative trauma disorders of the upper extremity in computer users: practice models based on systematic review. Work 2012;42:153-72

24 Lowry V, Desjardins-Charbonneau A, Roy J, et al. Efficacy of workplace interventions for shoulder pain: a systematic review and meta-analysis. J Rehabil Med 2017;49:529-42.

25 Richardson A, McNoe B, Derrett S, et al. Interventions to prevent and reduce the impact of musculoskeletal injuries among nurses: a systematic review. Int J Nurs Stud 2018;82:58-67.

26 Kelly D, Shorthouse F, Roffi V, et al. Exercise therapy and workrelated musculoskeletal disorders in sedentary workers. Occup Med 2018;68:262-72.

27 Stock SR, Nicolakakis N, Vezina N, et al. Are work organization interventions effective in preventing or reducing work-related musculoskeletal disorders? A systematic review of the literature. Scand J Work Environ Health 2018;44:113-33.

28 Crawford JO, Laiou E, Spurgeon A, et al. Musculoskeletal disorders within the telecommunications sector-A systematic review. Int $J$ Ind Ergon 2008;38:56-72.
29 Hoe VCW, Urquhart DM, Kelsall HL, et al. Ergonomic design and training for preventing work-related musculoskeletal disorders of the upper limb and neck in adults. Cochrane Database Syst Rev 2012;10.

30 Freiberg A, Euler U, Girbig M, et al. Does the use of small AIDS during patient handling activities lead to a decreased occurrence of musculoskeletal complaints and diseases? A systematic review. Int Arch Occup Environ Health 2016;89:547-59.

31 Hegewald J, Berge W, Heinrich P, et al. Do Technical Aids for Patient Handling Prevent Musculoskeletal Complaints in Health Care Workers? -A Systematic Review of Intervention Studies. Int J Environ Res Public Health 2018;15:476.

32 Verbeek JH, Martimo K-P, Karppinen J, et al. Manual material handling advice and assistive devices for preventing and treating back pain in workers. Cochrane Database Syst Rev 2011;25.

33 Rautiainen R, Lehtola MM, Day LM, et al. Interventions for preventing injuries in the agricultural industry. Cochrane Database Syst Rev 2008;96.

34 van der Molen HF, Basnet P, Hoonakker PLT, et al. Interventions to prevent injuries in construction workers. Cochrane Database Syst Rev 2018;48.

35 Cashman CM, Ruotsalainen JH, Greiner BA, et al. Alcohol and drug screening of occupational drivers for preventing injury. Cochrane Database Syst Rev 2009;39.

36 Mischke C, Verbeek JH, Saarto A, et al. Gloves, extra gloves or special types of gloves for preventing percutaneous exposure injuries in healthcare personnel. Cochrane Database Syst Rev 2014;30.

37 Parantainen A, Verbeek JH, Lavoie MC, et al. Blunt versus sharp suture needles for preventing percutaneous exposure incidents in surgical staff. Cochrane Database Syst Rev 2011;11.

38 Reddy VK, Lavoie M-C, Verbeek JH, et al. Devices for preventing percutaneous exposure injuries caused by needles in healthcare personnel. Cochrane Database Syst Rev 2017;54.

39 Verbeek JH, ljaz S, Mischke C, et al. Personal protective equipment for preventing highly infectious diseases due to exposure to contaminated body fluids in healthcare staff. Cochrane Database Syst Rev 2016;2.

40 Lunt JA, Sheffield D, Bell N, et al. Review of preventative behavioural interventions for dermal and respiratory hazards. Occup Med 2011;61:311-20.

41 Luong Thanh BY, Laopaiboon M, Koh D, et al. Behavioural interventions to promote workers' use of respiratory protective equipment. Cochrane Database Syst Rev 2016;12.

42 Bauer A, Rönsch H, Elsner P, et al. Interventions for preventing occupational irritant hand dermatitis. Cochrane Database Syst Rev 2018;55.

43 Tikka C, Verbeek JH, Kateman E, et al. Interventions to prevent occupational noise-induced hearing loss. Cochrane Database Syst Rev 2017;158.

44 Mischke C, Verbeek JH, Job J, et al. Occupational safety and health enforcement tools for preventing occupational diseases and injuries. Cochrane Database Syst Rev 2013;20.

45 van Vilsteren M, van Oostrom SH, de Vet HCW, et al. Workplace interventions to prevent work disability in workers on sick leave. Cochrane Database Syst Rev 2015;32.

46 Elsler D, Takala J, Remes J. An international comparison of the cost or work-related accidents and illnesses. Bilbao, Spain: European Agency for Safety and Health at Work, 2017.

47 Piso B, Semlitsch T, Reinsperger I, et al. Practical experience with overviews of reviews-valuable decision aid or academic exercise? Zeitschrift für Evidenz, Fortbildung und Qualität im Gesundheitswesen 2015;109:300-8.

48 Irving M, Eramudugolla R, Cherbuin N, et al. A critical review of grading systems: implications for public health policy. Eval Health Prof 2017;40:244-62.

49 Zhang Z, Xu X, Ni H. Small studies may overestimate the effect sizes in critical care meta-analyses: a meta-epidemiological study. Critical Care 2013;17.

50 Egger M, Smith GD, Schneider M, et al. Bias in meta-analysis detected by a simple, graphical test. BMJ 1997;315:629-34.

51 Balshem H, Helfand M, Schünemann HJ, et al. Grade guidelines: 3. rating the quality of evidence. J Clin Epidemiol 2011;64:401-6. 